\title{
Azithromycin and Reported Increased Risk of Cardiovascular Death
}

\author{
Kelly N. Koenig* and Craig G. Burkhart \\ College of Medicine, University of Toledo, 3000 Arlington Avenue, Toledo, OH 43614, USA
}

\begin{abstract}
Contrary to previous reports, azithromycin has now been associated with arrythmias and sudden cardiac deaths. There is a need for careful consideration of these risk factors with this drug especially when prescribing for the elderly. A more thorough screening of cardiovascular risk prior to starting azithromycin may also be warranted.
\end{abstract}

Keywords: Azithromycin, cardiac death, arrythmias.

\section{INTRODUCTION}

Azithromycin has been shown to be an effective treatment for several common dermatological problems, including skin infections, acne, and rosacea. Azithromycin had been reported to be relatively free of cardiotoxic effects, unlike related macrolides erythromycin and clarithromycin, which have been shown to increase the risk for ventricular arrhythmias and sudden cardiac death. Several reports of potential proarrythmic effects of azithromycin spurred further investigation. Recent findings published in the New England Journal of Medicine prompt careful consideration of the necessity for treatment with azithromycin, particularly in patients with a high baseline risk of cardiovascular disease.

Ray et al. [1] conducted a retrospective cohort study from patients enrolled in the Tennessee Medicaid program prescribed azithromycin (approximately 350,000 prescriptions) compared to matched persons who took no antibiotics, and patients who took amoxicillin, ciprofloxacin, or levofloxacin between 1992 and 2006. Participants were excluded if they exhibited serious noncardiovascular illness or had been hospitalized recently, and the resulting cohort ranged in age from 30 to 74 years old. Azithromycin use in this population was primarily for minor ear, nose, or throat infections and respiratory infections, similar to the indications for amoxicillin use. Patients taking a 5-day course of azithromycin compared to those who took no antibiotics were shown to have an increased risk of cardiovascular death (hazard ratio $[\mathrm{HR}], 2.88$ ) and death from any cause (HR, 1.85). Compared to amoxicillin, azithromycin was also shown to have an increased risk of cardiovascular death $(\mathrm{HR}, 2.49)$ and death from any cause (HR, 2.02). Azithromycin's use compared to amoxicillin's use was associated with an additional 47 cardiovascular deaths per 1 million courses; patients in the highest decile of cardiovascular disease risk had approximately 245 additional cardiovascular deaths per 1 million courses. Compared to the fluoroquinolones, azithromycin did demonstrate significantly greater risk of cardiovascular death than ciprofloxacin, but it

*Address correspondence to this author at the College of Medicine, University of Toledo, 3000 Arlington Avenue, Toledo, OH 43614, USA; Tel: 1-419890-7798; E-mail: kelly.koenig@rockets.utoledo.edu did not differ significantly from levofloxacin, which has known arrhythmic potential. The authors concluded that azithromycin use is associated with a small absolute increase in cardiovascular deaths, particularly in patients with a high baseline risk of cardiovascular disease [1].

Given the widespread use of azithromycin, a relative amount of correspondence followed this noteworthy study [2]. Several writers suggest that confounding cannot be completely resolved given the retrospective nature of the study. For instance, patient characteristics not assessed in the study and indication for antibiotic use might contribute to the increased risk of cardiovascular death. The authors counter that careful statistical analysis was utilized to control these factors, and that in particular, the indications for azithromycin and amoxicillin were similar (primarily for minor ear, nose, or throat infections and respiratory infections). In addition, two correspondence letters describe randomized, placebo-controlled trials that did not demonstrate an increased risk of cardiovascular death. Two such studies involving 11,759 patients with stable coronary artery disease showed no increase in mortality with $600 \mathrm{mg}$ of weekly azithromycin for 3 or 12 months [3, 4]. A third randomized, placebo-controlled trial involving 1,142 participants with chronic obstructive pulmonary disease (COPD) showed that daily azithromycin's use for 1 year reduced the risk of acute exacerbations of COPD [5]. This study did not demonstrate prolonged corrected QT interval (QTc), increases in death, or adverse cardiac events. However, $6 \%$ of candidates were excluded from the study for having a QTc interval greater than $450 \mathrm{msec}$, taking medications that prolong the QTc interval, for having a resting heart rate greater than 100 beats per minute, or for having a history of hypokalemia, congestive heart failure, or a family history of prolonged QTc interval. Furthermore, 10 patients were withdrawn from the study one month after randomization for prolonged QTc interval. The authors counter these 3 randomized, controlled studies by demonstrating that there was significantly less azithromycin exposure, totaling 955 person-years in all 3 studies, than the 4764 person-years in their retrospective study. Furthermore, the authors in the third randomized, controlled study described carefully excluded patients who might be susceptible to the proarrythmic effects of azithromycin [2]. 
In summary, despite the aforementioned potential weaknesses of this retrospective cohort study, it appears that azithromycin carries a higher degree of cardiovascular risk than previously thought. As with institution of any medication, the benefits to the patient must clearly outweigh the potential risks. Perhaps there is a role for more thorough screening of cardiovascular risk prior to starting azithromycin as a dermatologic therapy. This may, for instance, have a greater role in the typically older rosacea patients than the typical adolescent acne patient. In patients who demonstrated cardiovascular risk factors with clear indications for azithromycin, there may be a role for ECG screening for prolonged QTc interval. Regardless, further studies are necessary to further depict the potential proarrythmic risk of azithromycin and any role for such screening in patients prior to initiation of therapy.

\section{CONFLICT OF INTEREST}

The authors confirm that this article content has no conflicts of interest.

\section{ACKNOWLEDGEMENTS}

Declared none.

\section{REFERENCES}

[1] Ray WA, Murray KT, Hall K, Arbogast PG, Stein CM. Azithromycin and the risk of cardiovascular death. N Engl J Med 2012; 366: 1881-90.

[2] Correspondence to: Azithromycin and the risk of cardiovascular death. N Engl J Med 2012; 367: 772-5.

[3] Grayston JT, Kronmal RA, Jackson LA, et al. Azithromycin for the secondary prevention of coronary events. N Engl J Med 2005; 352: $1637-45$.

[4] O'Connor CM, Dunne MW, Pfeffer MA, et al. Azithromycin for the secondary prevention of coronary heart disease events: the WIZARD Study: a randomized controlled trial. JAMA 2003; 290: 1459-66.

[5] Albert RK, Connett J, Bailey WC, et al. Azithromycin for prevention of exacerbations of COPD. N Engl J Med 2011; 365:689-698 [Erratum, N Engl J Med 2012; 366: 1356]. 